\title{
Hereditary Neurodegenerative Disorder
}

National Cancer Institute

\section{Source}

National Cancer Institute. Hereditary Neurodegenerative Disorder. NCI Thesaurus. Code C97073.

An inherited disorder characterized by progressive degeneration and atrophy of the nervous system. 